\title{
Adipose tissue fatty acid and lipid mediator composition in obesity and response to chronic marine omega-3 fatty acid supplementation
}

\section{Abstract}

Introduction: Obesity is an excess of adipose tissue (AT) and is linked with increased inflammation that enhances risk of type-2 diabetes and cardiovascular disease. The BIOCLAIMS study assessed the fatty acid (FA) and lipid mediator composition in normal weight and obese individuals, and their response to chronic omega-3 FA supplementation.

Materials and methods: AT biopsies were collected pre- and post-12 week supplementation with $1.1 \mathrm{~g}$ EPA $+0.8 \mathrm{~g}$ DHA/day or corn oil. The composition of FA in the total lipid extract (TLE) of AT from 37 normal weight and 44 obese subjects was assessed by gas chromatography (GC) and the concentration of lipid mediators in AT TLE of 36 normal weight and 45 obese subjects by coupled GC-mass spectrometry.

Results: AT of obese subjects had higher concentrations of arachidonic acid (AA), EPA, DPA, PGF2 $\alpha$, arachidonoylethanolamine (AEA), and eicosapentaenoylethanolamine (EPEA) than that of normal weight subjects $(P<0.05)$. Obese subjects also had and lower concentrations of mediators derived from linoleic and $\alpha$-linolenic acids, DHA-derived mediators including RvD2 and hydroxydocosahexaenoic acids (HDHAs), AA-derived mediators including lipoxin-B4, hepoxilin-A3, 11,12-dihydroxyeicosatetrienoic acid (DHET), and 6-keto-PGF1 $\alpha$, and the FA ethanolamine of myristic acid and glycerol ester of palmitic acid $(P<0.05$ all $)$ than normal weight subjects.

Chronic supplementation with EPA + DHA increased the concentration of EPA $(P=0.006)$ and EPA-derived lipid mediators including dihydroxyeicosatetrienoic acids, EPEA, and EPA-glycerol ester $(P<0.05)$, and increased the concentration of DHA $(P$ $<0.001)$, docosahexaenoylethanolamine, and 8-, 11-, 14-, and 16-HDHA $(P<0.05)$. Chronic supplementation with EPA + DHA also decreased the concentration 4-, 17-, and 20-HDHA, and AA-derived lipid mediators including DHETs, AEA, and LTs in normal weight subjects $(P<0.05)$, and decreased $2-\mathrm{AG}$ in obese subjects $(P<0.05)$.

Discussion: These data indicate altered lipid signalling in AT in obesity (at baseline) suggesting dysregulation of adipose tissue expansion and inflammatory signalling, including lack of self-resolution, as well as dysregulation in the utilization of supplementary EPA + DHA for synthesis of anti-inflammatory lipid mediators. EPA + DHA are able to modulate synthesis of EPA-, DHA- and AA-derived lipid mediators but obesity may involve resistance to these effects particularly in endocannabinoid synthesis.

\section{Conflict of Interest}

There is no conflict of interest 